\title{
Short communication: Rapid antibiotic screening tests detect antibiotic residues in powdered milk products
}

\author{
J. Kneebone, P. C. W. Tsang, and D. H. Townson ${ }^{1}$ \\ Department of Molecular, Cellular, and Biomedical Sciences, University of New Hampshire, Durham 03824
}

\begin{abstract}
Rapid antibiotic screening tests are widely used in the dairy industry to monitor milk for the presence of antibiotic residues above regulated levels. Given the persistent concern over contamination of milk products with antibiotic residues, we investigated the utility of IDEXX Snap test devices (IDEXX Laboratories Inc., Westbrook, ME) as tools for detecting antibiotic residues in powdered milk products. Five powdered milk products were reconstituted according to manufacturer specification with distilled water: Carnation (Nestlé USA Inc., Solon, OH), Nido youth and Nido adult (Nestlé Mexico Inc., Mexico City, Mexico), ELK (Campina, Eindhoven, the Netherlands), and Regilait (Saint-Martin-Belle-Roche, France). Positive samples were generated by spiking reconstituted milk with penicillin G, cephapirin, or tetracycline to either the European Union-regulated maximum residue limit or the FDA-regulated safe/tolerance level, whichever was lower. Control, unspiked negative milk samples and positive samples were tested with appropriate IDEXX Snap test kits (penicillin G and cephapirin with New Beta-Lactam, tetracycline with New Tetracycline). All samples yielded definitive results consistent with expectations, and there were no instances of false-positive or false-negative readings. These results suggest that both the New Beta-Lactam and New Tetracycline IDEXX Snap test kits effectively detect antibiotic residues in commercially available powdered milk samples and are useful tools for monitoring antibiotic residues in reconstituted powdered milk products.
\end{abstract}

Key words: powdered milk, IDEXX Snap test, antibiotic residue

International trade of dry or powdered milk products is a multibillion dollar industry. From 2003 to 2008, an average of 2.5 million tonnes of whole powdered milk and nonfat dry milk was exported annually worldwide (USDA, 2008). With global demand for dairy products

Received January 5, 2010.

Accepted May 19, 2010.

${ }^{1}$ Corresponding author: dave.townson@unh.edu increasing annually, production and exportation of dry or powdered milk products or both will continue to increase, as will the need to monitor those products for potentially hazardous substances.

The widespread use of various classes of antibiotics within the dairy industry has led to increased concern over consumption of contaminated milk products and associated human health risks. Although establishment of various safeguards (e.g., maximum residue limits) have facilitated more effective monitoring of commercial milk product quality, recent studies suggest that antibiotic residue contamination is still problematic in some countries (e.g., Brazil: Martins-Júnior et al., 2007; Bando et al., 2009; China: Bai et al., 2005; Bai and Huang, 2006; Kenya: Shitandi and Sternesjö, 2004). Interestingly, most studies on antibiotic residue testing have focused on fluid milk and little attention has been paid to powdered milk products. Given the growing demand for milk products and the persistent threat of antibiotic contamination, the need to monitor imports of powdered milk products for a variety of potentially harmful substances is of utmost importance.

The use of commercially available antibiotic residue screening tests has played a large role in preventing the unintentional sale and consumption of antibioticcontaminated fluid milk products. These tests, originally designed for producing rapid on-farm results, have proven to be effective tools for detecting antibiotic residues in commingled milk from several species (Contreras et al., 1997; Andrew, 2000). Presently, tests are available to monitor for several antibiotics in fluid milk, including aminoglycosides (e.g., gentamicin), $\beta$-lactams (e.g., penicillin $\mathrm{G}$, cephapirin), sulfonamides (e.g., sulfamethazine), and the tetracyclines. However, no commercially available rapid tests to screen for antibiotic residues are approved for powdered milk products. Moreover, the compatibility of these tests with reconstituted powdered milk has yet to be examined.

Building upon the utility of rapid screening tests for antibiotic residues in fluid milk, we were interested in determining whether these tests are similarly effective with powdered milk products. Specifically, our objectives were to examine whether IDEXX Snap tests (IDEXX Laboratories Inc., Westbrook, ME) detect antibiotic 
residues in 5 randomly selected, commercially available powdered milk products: Carnation (Nestlé USA Inc., Solon, OH), NIDO youth and NIDO adult (Nestlé Mexico Inc., Mexico), ELK (Campina, Eindhoven, the Netherlands), and Regilait (France). We hypothesized that these tests would be capable of detecting penicillin $\mathrm{G}$, cephapirin, and tetracycline in reconstituted powdered milk products when spiked at regulated levels.

All powdered milk products were reconstituted on the day of testing according to the manufacturer's specification using distilled water. To ensure thorough reconstitution, all samples were mixed well by inversion and kept on ice or at $4^{\circ} \mathrm{C}$ for at least $1 \mathrm{~h}$ before testing. The lyophilized antibiotics were obtained from the United States Pharmacopeia (Rockville, MD) and reconstituted according to the manufacturer's guidelines. Antibiotic stock solutions were prepared as follows: penicillin $\mathrm{G}$ and cephapirin at $1,000 \mu \mathrm{g} / \mathrm{kg}$ in $10 \mathrm{mM}$ PBS (pH 7.2) and tetracycline at $5,000 \mu \mathrm{g} / \mathrm{kg}$ in $5 \mathrm{mM}$ sodium citrate ( $\mathrm{pH} 3.0)$. Prepared solutions were stored at $4^{\circ} \mathrm{C}$ for the duration of the study.

All milk preparations were individually spiked to either the European Union-regulated maximum residue limit or the FDA-regulated safe/tolerance level, whichever was lower for several of the most widely used antibiotics (Table 1). For the reconstituted milk powders, 10 unspiked samples (negative controls) and 10 spiked samples at regulated levels were tested for each antibiotic. All samples were tested with the appropriate IDEXX Snap test kit (penicillin G and cephapirin with New Beta-Lactam and tetracycline with New Tetracycline). On each day of testing, fresh commingled milk from the University of New Hampshire Fairchild Dairy Teaching and Research Center (Durham) was simultaneously tested with 4 samples as known negative milk and 4 samples spiked at the regulated level with antibiotic. All samples were numerically coded for randomization before testing. Test kits were used per manufacturer's instructions and the samples were analyzed and recorded using the IDEXX Snapshot Reader (IDEXX Laboratories Inc.).

Testing of each antibiotic was completed over the course of 1 to $3 \mathrm{~d}$ (tetracycline: $3 \mathrm{~d}$; penicillin G: $2 \mathrm{~d}$; ce-

Table 1. European Union maximum residue limit (EU-MRL) and FDA safe/tolerance level for antibiotics $(\mu \mathrm{g} / \mathrm{kg})$

\begin{tabular}{lcc}
\hline Antibiotic & EU-MRL & $\begin{array}{c}\text { FDA safe/ } \\
\text { tolerance level }\end{array}$ \\
\hline Tetracycline & $100^{1}$ & 300 \\
Penicillin & $4^{1}$ & 300 \\
Cephapirin & 20 & 60 \\
\hline
\end{tabular}

${ }^{1}$ Reconstituted milk powders were spiked with antibiotic at the lower of the 2 levels. phapirin: $1 \mathrm{~d}$ ). All reconstituted milk samples migrated through the SNAP test device and provided a definitive outcome. Individual migration rates, however, varied considerably between the powdered milk products. Most preparations, including commingled milk, reached the activation circles on the Snap test device within 1 to 2 min after application. The exception was the preparation of Nido youth, which required migration times of up to $8 \mathrm{~min}$. This preparation appeared visually more viscous than any of the other products, suggesting that its slow migration through the test device was likely a result of its viscosity. Although the observed differences in migration rates of individual powder milk preparations were a concern, they did not affect the sensitivity of the Snap tests. The devices provided results consistent with the sample type in every instance.

Positive and negative results generated by the IDEXX Snapshot Reader were accompanied by a numerical ratio corresponding to the intensity of the control spot relative to the intensity of the sample spot. The control:sample ratios ranged from 0.25 to 1.05 and from 1.93 to $>10.0$ for negative and positive results, respectively (Table 2). There were no instances of falsepositive or false-negative readings. For all trials, the Snapshot Reader results were in agreement with the known identity of the blinded samples. In 4 instances (i.e., 4/352 test devices), the initial reading of the Snap test device yielded an invalid result. Upon subsequent rereading of the device, however, the outcome was a definitive positive result consistent with the type of sample. The initial invalid reading in these 4 instances was not associated with a particular antibiotic or type of reconstituted milk sample.

The ability of the New Beta-Lactam and New Tetracycline IDEXX Snap tests to effectively detect regulated levels of antibiotic residue in commercially available powdered milk samples demonstrates their potential utility for rapid screening of these products. In every trial, the results were consistent with what was expected because all negative samples tested negative and all positive samples tested positive. These results provide encouraging evidence that both of these rapid screening tests are potentially useful tools for monitoring powdered milk products for the presence of antibiotic residues.

Several studies have raised concerns about the utility of rapid on-farm antibiotic screening tests because of a high occurrence of false-positive results (Cullor, 1992; Tyler et al., 1992; Van Eenennaam et al., 1993; Norell et al., 1994; Andrew et al., 1997; Andrew, 2000). Incidences of false positives in raw milk tests have been correlated to several factors including high levels of lactoferrin, lysozyme, milk fat, milk protein, and SCC (Carlsson et al., 1989; Van Eenennaam et al., 1993; 
Table 2. Results [average \pm SD (range)] generated by the IDEXX SNAPshot Reader for all powdered milk products and antibiotics ${ }^{1}$

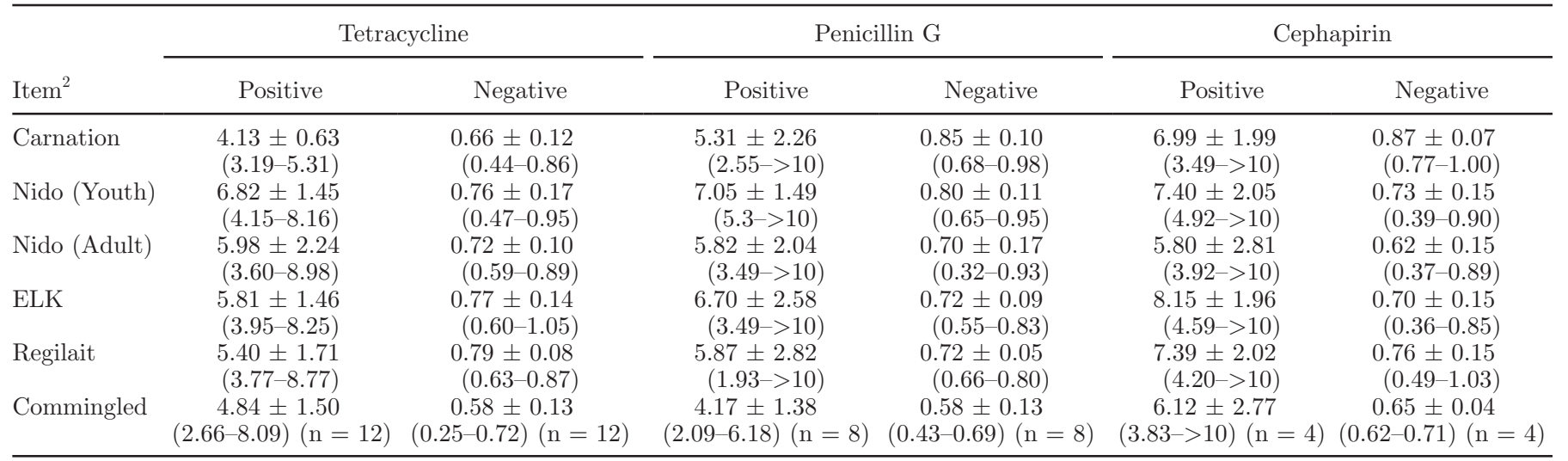

${ }^{1}$ Results are displayed as mean control:sample (C:S) spot ratios and SD for both positive (spiked; $\mathrm{n}=10$ ) and negative (unspiked; $\left.\mathrm{n}=10\right)$ reconstituted powdered milk samples and commingled milk (presented with corresponding sample numbers). C:S ratios from 0.25 to $1.05=$ negative result; C:S ratios from 1.93 to $10=$ positive result. C:S ratios $>10$ were rounded to 10 for all calculations of mean and SD.

${ }^{2}$ Carnation: Nestlé USA Inc. (Solon, OH); Nido youth and Nido adult: Nestlé Mexico Inc. (Mexico); ELK: Campina (Eindhoven, the Netherlands); Regilait: (France).

Andrew, 2000). Interestingly, performance of antibiotic residue test devices may vary between species. According to Andrew (2000), there was a trend toward increased false-positive outcomes when screening tests were used to evaluate milk from Jersey cows compared with testing milk from Holstein cows. With respect to IDEXX Snap tests, false-positive readings were associated with increased milk fat. Alternatively, Contreras et al. (1997) did not record any false-positive results when testing caprine milk for antibiotic residues using bovine Snap test devices and, overall, had great success applying bovine screening tests to caprine milk. The lack of any false-positive results in the current study is encouraging and suggests that IDEXX Snap New Beta-Lactam and New Tetracycline test devices are effective tools for detecting antibiotic residues in powdered milk.

All samples tested ran successfully in the devices and generated definitive results that corresponded to the expected outcome. The results of this study suggest that both New Beta-Lactam and New Tetracycline IDEXX Snap test devices are capable of detecting antibiotic residues at regulated levels in various reconstituted powdered milk products. Further work is needed to determine the compatibility of these tests with a wider range of powdered milk products (e.g., infant formulas) as well as the utility of other commercially available screening tests with powdered milk products. Nevertheless, we are optimistic that this technology will prove to be an effective tool for monitoring antibiotic residues in powdered milk products.

\section{ACKNOWLEDGMENTS}

This work was supported in part by the New Hampshire Agricultural Experiment Station (Durham) and is scientific contribution number 2421. The authors thank Travis Waldron (IDEXX Laboratories) and IDEXX Laboratories Inc. (Westbrook, ME) for providing the IDEXX Snap test kits and resources for this study.

\section{REFERENCES}

Andrew, S. M. 2000. Effect of fat and protein content of milk from individual cows on the specificity rates of antibiotic residue screening tests. J. Dairy Sci. 83:2992-2997.

Andrew, S. M., R. A. Frobish, M. J. Paape, and L. J. Maturin. 1997. Evaluation of selected antibiotic residue screening tests for milk from individual cows and examination of factors affecting the probability of false positive outcomes. J. Dairy Sci. 80:30503057.

Bai, J., and S. Huang. 2006. Assay on residue of antibiotics in pure milk. J. Dairy Sci. Tech. (China) 2006-01.

Bai, J., W. Liang, H. Sun, J. Cao, and S. Huang. 2005. Examination of residue of antibiotics in pure milk from retail market. Bev. Fast Froz. Food Ind. (China) 11:34-37.

Bando, E., R. C. Oliveira, G. M. Ferreira, and M. Machinski Jr. 2009. Occurrence of antimicrobial residues in pasteurized milk commercialized in the state of Paraná, Brazil. J. Food Prot. 72:911-914.

Carlsson, A., L. Bjorck, and K. Persson. 1989. Lactoferrin and lysozyme in milk during acute mastitis and their inhibitory effect in Delvotest P. J. Dairy Sci. 72:3166-3175.

Contreras, A., M. J. Paape, A. L. DiCarlo, R. H. Miller, and P. Rainard. 1997. Evaluation of selected antibiotic residue screening tests for milk from individual goats. J. Dairy Sci. 80:1113-1118.

Cullor, J. S. 1992. Cowside testing for antibiotic residues: Problems and solutions. Pages 153-159 in Proc. 31st Ann. Mtg. Natl. Mastitis Counc., Arlington, VA. Natl. Mastitis Counc. Inc., Arlington, VA. 
Martins-Júnior, H. A., T. A. Kussumi, A. Y. Wang, and D. T. Lebre. 2007. A rapid method to determine antibiotic residues in milk using liquid chromatography coupled to electrospray tandem mass spectrometry. J. Braz. Chem. Soc. 18:397-405.

Norell, R. J., J. H. Packham, and L. K. Fox. 1994. Effect of clinical mastitis on antibiotic residue tests. Pages 377-378 in Proc. 33rd Ann. Mtg. Natl. Mastitis Counc., Orlando, FL. Natl. Mastitis Counc. Inc., Arlington. VA.

Shitandi, A., and A. Sternesjö. 2004. Factors contributing to the occurrence of antimicrobial drug residues in Kenyan milk. J. Food Prot. 67:399-402.
Tyler, J. W., J. S. Cullor, R. J. Erskine, W. L. Smith, J. Dellinger, and K. McClure. 1992. Milk antimicrobial drug residue assay results in cattle with experimental, endotoxin-induced mastitis. J. Am. Vet. Med. Assoc. 201:1378-1384.

USDA. 2008. Dairy: World markets and trade. Circular series, FD 1-08, July 2008. USDA, Washington, DC.

Van Eenennaam, A. L., J. S. Cullor, L. Perani, I. A. Gardner, W. L. Smith, J. Dellinger, and W. M. Guterbock. 1993. Evaluation of milk antibiotic residue screening tests in cattle with naturally occurring clinical mastitis. J. Dairy Sci. 76:3041-3053. 